\title{
GPS/BDS relative positioning assessment by zero baseline observation
}

\author{
Xu Tang ${ }^{1 *}$, Gethin Wyn Roberts ${ }^{2}$, Craig Matthew Hancock ${ }^{1}$, Jianjun $\mathrm{Yu}^{3}$
}

Xu.Tang@nottingham.edu.cn

1. University of Nottingham Ningbo, 199 Taikang East Road, Ningbo, 315100, China

2. The University of the Faroe Islands, Faroe Islands.

3. GEOMATICS CENTER OF ZHEJIANG, 83 North Baochu Road, Xihu District, Hangzhou, 310012, China

\begin{abstract}
A lot of researches proved integration of GPS and BDS could improve the position precision, since the Chinese BDS was opened to the Asia-Pacific users. Does this improvement come from better geometry by combining GPS/BDS or more precise measurement applied? The studies which have been focused on could be summarized in: how the integration system improved the reality ambiguity resolution; the advantage of integration by enhance the number of tracked satellites during high cut-off elevation, etc. In this paper, zero baseline observation was employed to assess the GPS/BDS standalone and integration system positioning precision without the effection of multipath, ionospheric and tropospheric delays, etc. DOP was used a lot to assess the geometry of GNSS satellites from previous research. GNSS positioning precision is not only determined by the precision of measurement, but also satellites geometry. This study presents the position error amplify indicator and variance amplify indicator to assess the geometry and carrier phase measurement contributions for the position precision improvement in GPS/BDS standalone and integration system applications. Additionally, difference level of random noise was simulated based on the real carrier phase measurements. North, east and up component of GPS/BDS standalone and integration systems' position precision reduced by the increased noise of simulated measurement, but have different characteristic.
\end{abstract}

Keywords: position precision assessment; Chinese BeiDou satellite navigation; GPS; zero baseline

\section{Introduction}

Chinese satellite navigation system called BDS, also known as BeiDou is working in the great Asia-Pacific area. A lot of researches about BDS have been doing so far, e.g. orbit and clock determination (Zhao et al. 2013; F. He et al. 2014; B. Wang et al. 2015; Zhang et al. 2015), the impact of BDS measurements bias for positioning and orbit determination (Nadarajah et al. 2015; Gu et al. 2015; Guo, Zhang, and Wang 2015; Yinhua et al. 2015), etc. The earlier days, researches were involved to simulate the BeiDou constellation and analyzed the DOP distribution in the whole Asia-Pacific area (Chen et al. 2009; Chiang et al. 2010). After the initial assessment BDS positioning by BDS GEO and IGSO satellites(Shi et al. 2013; Montenbruck et al. 2013), a lot of integrating BDS and GPS, etc. studies have been investigated. These works confirmed that integration GPS and BDS 
could extend the applications in high cut-off elevation situations. Additionally, integrating GPS and BDS could improve the performance of relative positioning ambiguity resolution ( $\mathrm{H}$. He et al. 2014; Yang et al. 2014; Teunissen, Odolinski, and Odijk 2014; Odolinski, Teunissen, and Odijk 2015), as well as reducing the convergence time in precise point positioning (PPP) applications ( $L i$ et al. 2015). Single epoch ambiguity resolution based on the advantage of long wavelength linear combined carrier phase measurements is stable by integration GPS and BDS (Deng et al. 2014). This also implies that ambiguity strategy base on couples of linear combined measurements with different characteristic of noise, (e.g. Geometry-free Trip-Carrier Ambiguity Resolution (TCAR), Cascading Integer Resolution (CIR), etc.) is also benefited by integrating multi-GNSS.

Accuracy of the measurements directly reveal the noise level of positioning. BDS satellites have differently characteristic of measurement elevation dependent noise as the constellations is mixed by geostationary earth orbit (GEO) satellites, inclined geosynchronous orbit (IGSO) satellites and medium earth orbital (MEO)satellites. The difference of GEO/IGSO and MEO satellites' orbital period make the BDS positioning bias caused by multipath is complex. GPS positioning bias caused by multipath has very strong consistence with the GPS orbital period, which is different in BDS system. Mitigation of the BDS multipath delays on measurements by the repeat of satellites could effective improve the position precision(Ye et al. 2015; G. Wang et al. 2015). Elevation-dependent code variations are severely affected the PPP applications, but little effect in standard point positioning due to the accuracy of broadcast ephemerides. Multipath is unneglectable when assessing precise positioning. Zero baseline is the best option for assessing position precision, could simply get rid of the effection of multipath (Msaewe et al., 2017).

In this paper, we present a new method for assessing the satellite geometry, which could be used as revealing how much noise is amplified at the components of north, east and up; also, the GPS/BDS integration system positioning precision is improved by the combined constellation or the more precise carrier phase measurements. Couple of studies have been involved with the integration of GPS and BDS, as well as the precision assessment of the carrier phase measurements. Dilution of precision (DOP) has been the indication for assessing the quality of the satellite geometry so far. But, DOP cannot explain well how the system amplify the position noise with the same level noise of GPS and BDS observation, respectively. In this study, we found coefficient of GPS/BDS integration system is not always the optimal, comparing with that of GPS and BDS standalone, respectively. The coefficient quality of equations is not determined by more tracked satellites. Integration system positioning precision improved more determined by the more precise carrier phase measurements were used. With the controlled simulated noise in the zero baseline measurements, we found that the position noise at north and east component, by GPS-only and BDS-only respectively, increased with the same rate by the controlled simulated noise. Position noise at up component by GPS-only and BDS-only is different with each other. Besides, integrating GPS and BDS could significantly improve the position precision.

This manuscript is organized as follows. We firstly present position error serials and DOP by GPS-only, BDS-only and GPS/BDS integration system. Double differenced (DD) carrier phase measurements are also analyzed in section 2 . In section 3, position error and variance evaluation indicator for GPS/BDS integration and standalone positioning model, as well as its mathematic derivation. The reason of up component is always worse than horizontal is also given in this section. Besides, integration system positioning precision is improve by more precise measurements is applied, rather than the constellation geometry is improved. In section 4, GPS and BDS 
constellation include different types of satellites. Assistance with the different level of controlled noise measurements, the GPS/BDS integration and standalone position noise behavior at north, east and up components were presented in this section. All the work and conclusion were summarized in the last section.

\section{GPS/BDS zero baseline experiment}

Positioning precision is determined by not only the precision of measurements, but also its constellations and the distribution of navigation satellites. Satellite and receiver Clockers' errors could be absolutely estimate by double differencing; lonospheric and tropospheric delays could be significantly reduced in general GNSS baseline applications. Multipath is still the major error in the procedure of positioning assessment.

\subsection{Zero baseline field experiment}

Two ComNav K508 unhoused receivers were connected to one antenna using a GEMS signal splitter (PN: GS18). These two receivers have the capacity of tracking GPS L1, L2 and L5 signals, BDS B1, B2 and B3 signals, as well as the GLONASS signals. These two receivers tracked the satellite signals at the same antenna phase center; ionospheric, tropospheric and multipath delays are exactly same in the two receivers' measurements. Observations from the two receivers could be assumed as a baseline having a distance of "zero" units. The time series results of the final baseline processing reveals the precision of the two satellite navigation systems, as compared to the known distance of zero unites. 24 hours (one whole BDS orbital period, UTC 00:00:00-23:59:59) of data were collected on June $5^{\text {th }}$ 2014. A Leica AR 25 choke ring antenna was located on a pillar on the roof of the Faculty of Science and Engineering building, University of Nottingham Ningbo China (UNNC) (figure 1). The cable used to connect the Leica AR25 choke ring antenna and the signal splitter is about 50 meters; another two 3-meter antenna cables were used to connect the splitter with the two ComNav receivers. Both receivers' sampling rate and the cut-off angle were set as $1 \mathrm{~Hz}$ and 15 degree, respectively.

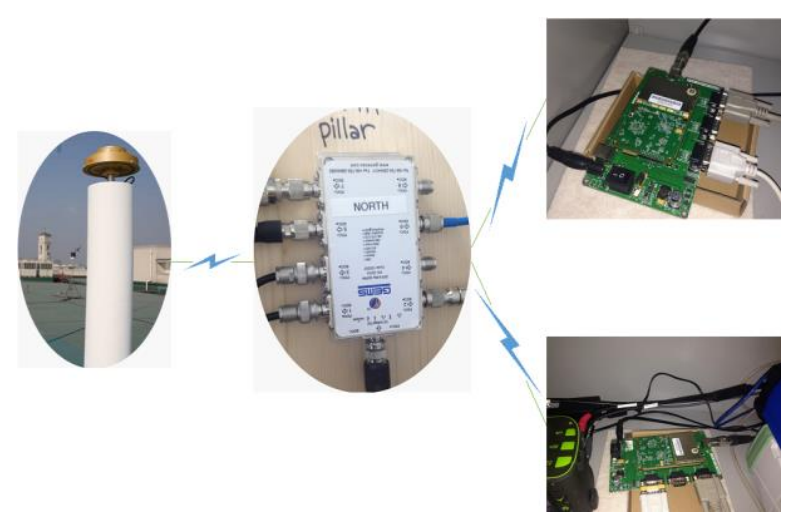

Fig. 1 Zero baseline field experiment; a Leica AR25 choke ring antenna, connected to the two ComNav K508 GNSS receivers via the GEMS signal splitter.

\section{2 zero baseline relative positioning model}

Two receivers marked as $i$ and $j$ are connected with an antenna by splitter. We also assume there are two tracked satellites named as $p$ and $q$. Considering about the tropospheric 
noise, ionospheric noise, multipath noise, satellite and receivers' clock errors, as well as the receivers random noise. The observation equation by receiver $i$ tracking satellite $p$ could be written as:

$$
\varphi_{i}^{p}=\rho_{i}^{p}-c\left(\delta t^{p}-\delta t_{i}\right)+N_{i}^{p}+T_{i}^{p}+I_{i}^{p}+M_{i}^{p}+\zeta_{i}^{p}
$$

Where $\varphi$ is the carrier phase measurement; $p$ and $i$ represent the tracked satellite and receiver, respectively; $\rho$ is the geometrical range between satellite and receiver; $c$ is light speed in vacuum; $\delta t^{p}$ is the satellite $p$ clock's error; $\delta t_{i}$ is the receiver $i$ clock's error; $N_{i}^{p}$ is the carrier phase integer ambiguity; $T_{i}^{p}$ and $I_{i}^{p}$ are the tropospheric and ionospheric delays, respectively; $M_{i}^{p}$ is the multipath delay of receiver $i$ from satellite $p ; \zeta_{i}^{p}$ is the carrier phase random noise. With getting involved another receiver $j$ and tracked satellite $q$, double differenced model could be written as:

$$
\varphi_{i, j}^{p, q}=\rho_{i, j}^{p, q}+N_{i, j}^{p, q}+T_{i, j}^{p, q}+I_{i, j}^{p, q}+M_{i, j}^{p, q}+\zeta_{i, j}^{p, q}
$$

Where, the general relationship $g_{i, j}^{p, q}=g_{j}^{q}-g_{j}^{p}-g_{i}^{q}+g_{i}^{p}$. With the assist of splitter, these two receivers tracked the same satellite by the only one antenna. Tropospheric, ionospheric and multipath errors could be absolutely eliminated. Eq.(2) could be rewritten as:

$$
\varphi_{i, j}^{p, q}=\rho_{i, j}^{p, q}+N_{i, j}^{p, q}+\zeta_{i, j}^{p, q}
$$

\section{3 zero baseline DD differenced carrier phase random errors}

$\zeta_{i, j}^{p, q}$ is the only errors left in double differenced measurement by the zero baseline dataset.

Fig. 2 showes both GPS (sat 20 and 28) and BDS (sat 3 and 11) DD carrier phase measurements time-error serials results. Both GPS and BDS DD measurements' random error are sensitive with satellites elevation when its elevation below 45 degree. In case of effection from elevationdependent error, mask-off elevation was set to 45 degree, Fig. 3 gives all the GPS and BDS DD measurements random error without the elevation-dependent noise during a whole BDS orbital period. The random noise serial reveals that both of GPS and BDS DD carrier phase measurements accuracy accesses millimeter level. The bottle right corner subfigure gives the GPS and BDS L1/L2/B1/B2/B3 DD measurements standard deviation. GPS L1 accuracy, with the smallest STD performs, is obviously better than that of GPS L2 and BDS triple frequencies. BDS B3 carrier phase accuracy performs slightly better than B2 and B1. 

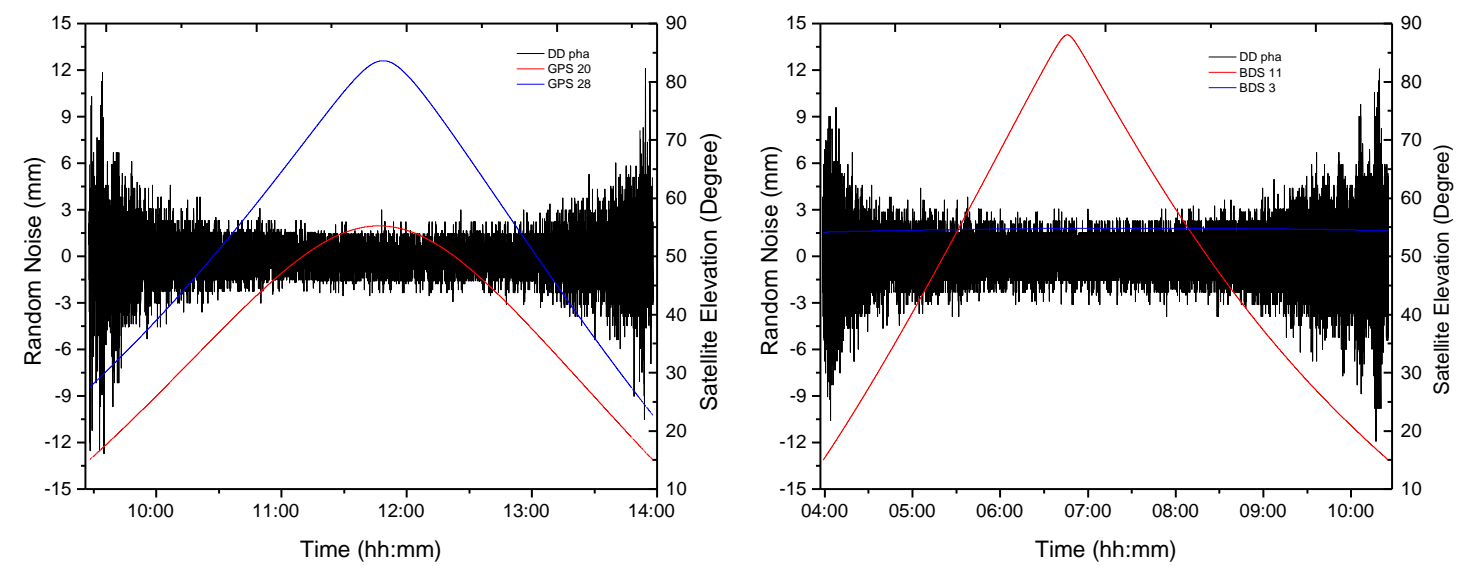

Fig. 2 error time serials of GPS and BDS double differenced carrier phase measurements (maskoff elevation: 15; GPS: left, BDS: right)
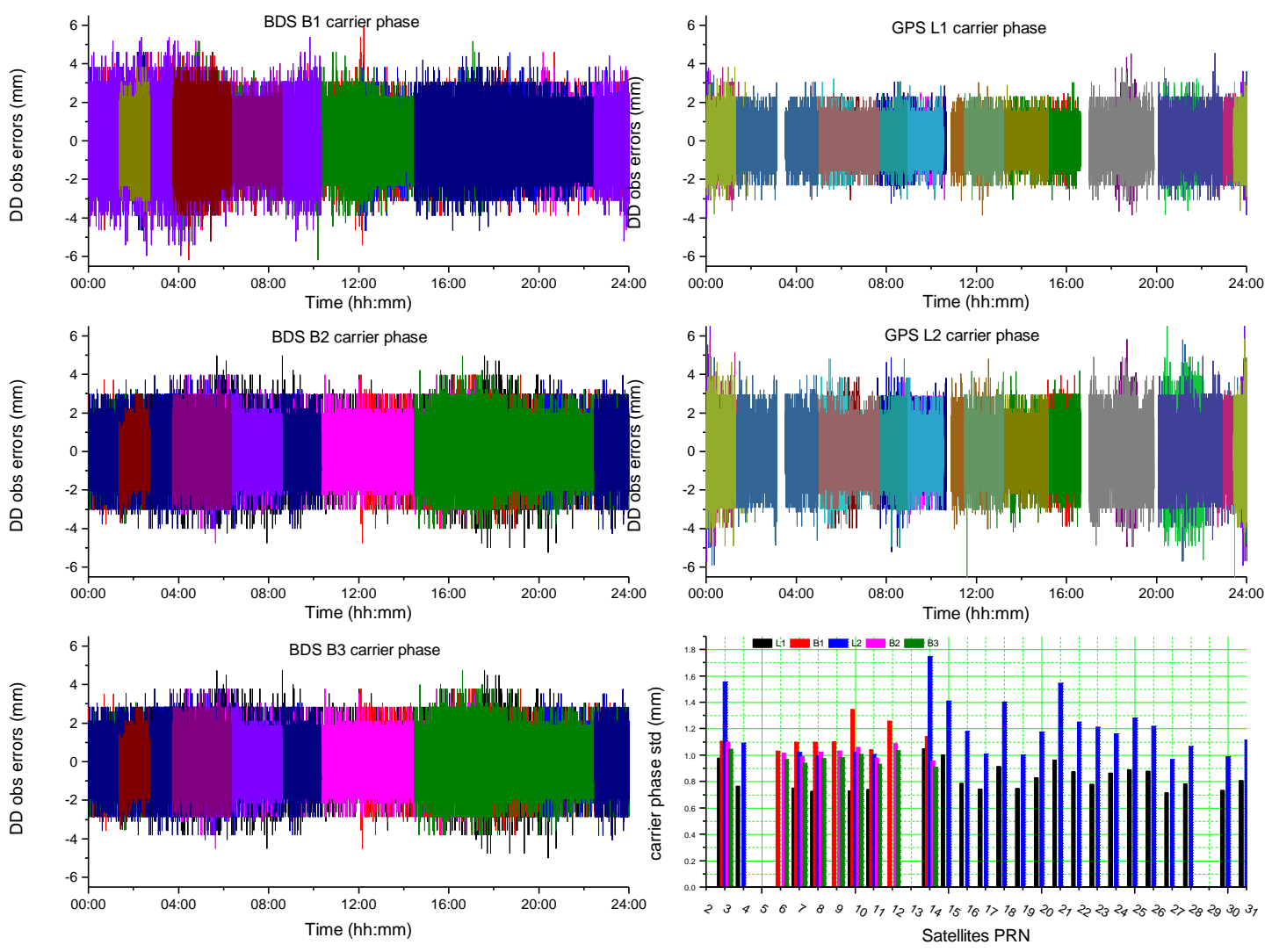

Fig. 3 GPS L1/L2 and BDS B1/B2/B3 double differenced carrier phase measurements random errors (mask-off angle: 45); GPS/BDS satellites' DD measurements standard deviation (std)

\section{4 relationship between the number of tracked satellites and its GDOP}

The number of satellites and DOP are usually used to assess the multi-GNSS, respectively. We collected 24 hours GPS/BDS measurements with the cut-off 15 degree angle, and recorded the number of tracked satellites and calculated GDOP.

Integrating GPS/BDS could enhance the number of tracked satellites, also reduces the GDOP during the 24 hours. Integration multi-GNSS has advantage in extreme situations. 


$$
G D O P=\operatorname{trace}\left\{\left(\mathrm{B}^{T} \mathbf{P B}\right)^{-1}\right\}
$$

$\mathrm{B}$ is the coefficient of point positioning coefficient. $\mathbf{P}$ is the weight during least square operator.

Figure 4 shows integration of GPS and BDS could improve GDOP all the time. The short period when only 4 GPS satellites tracked (background is marked green with alphabet $E$ in Figure 4), GPS GDOP is too big to be used for high precise positioning application. Integrating GPS and BDS is one of the solutions for this standalone GPS events. Period marked alphabet $A$ in figure 4 reveals that better GDOP is not only determined by more tracked satellites. There are more BDS could be tracked than GPS, but GPS GDOP performs better than that of BDS. Periods of $B, C$ and $D$ reveal that GDOP could be different with same number of tracked satellites (comparing between $C$ and D). Period $B$ and $C$ present that only 5 GPS satellites could give a better GDOP, comparing with the period that 8 to 9 GPS satellites could be tracked. GDOP is determined by the number of tracked satellites, but not the only factor. Period C and D GPS results in figure 4 reveal that the distribution of tracked satellites is also one of the factors effects the GDOP.

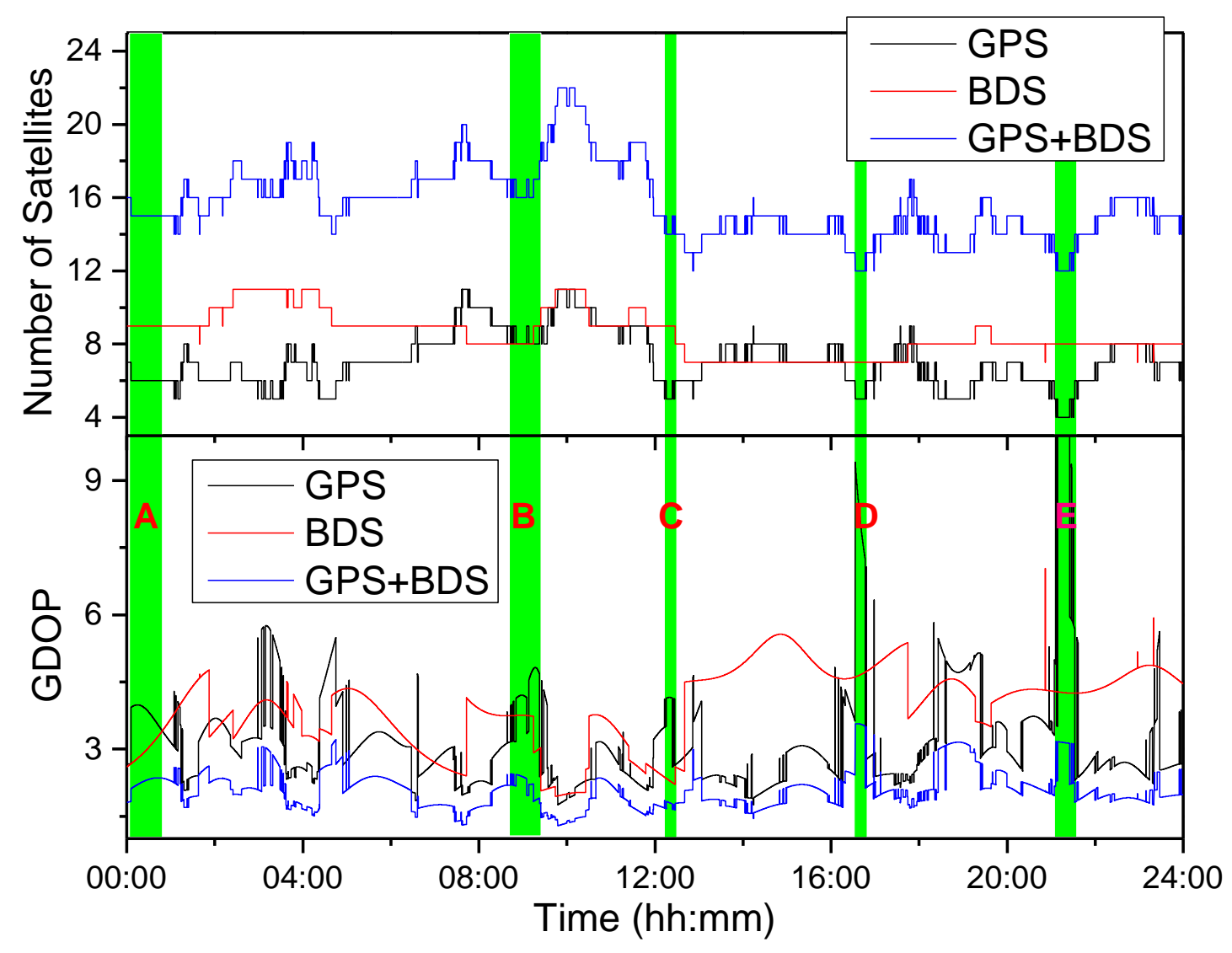

Fig. 4 Number of GPS/BDS tracked satellites and GDOPs during 24 hours (mask-off angle is 15 degree)

\section{5 improvement of positioning by integrating GPS and BDS}

In this subsection, we analysis the precision positioning contribution by integrating the GPS and BDS. GPS L1 is more precise than L2 (figure 3), positioning precision by L1/L2 is agree with the 
precision of measurement (figure 5 , upon panel). Figure 5 includes position errors at the components of north, east and up by GPS/BDS integration and standalone system, respectively. Zero baseline experiment test reveals BDS and GPS standalone system have singular point, which is improve by integration system (Fig. 5 bottle panel). Up component has much more noise than the component of east and north in GPS/BDS standalone and integration system. Bigger positioning errors from GPS system mostly caused by the small number of tracked satellites (see the figure 5 position error and Figure 4 around 21:00). The 24 hours' time-serial errors at the components of north, east and up demonstrates that the positioning error is obviously improved by integrating GPS and BDS. Table 1 gives the GPS/BDS standalone and integration system position standard deviation at three components at the cut off angle 15 degree and 30 degree. GPS position precision is better than GPS L2 and BDS B1/B2. GPS position precision is reduced obviously when the cut-off angle was set at 30 degree. Standard deviation of BDS position is slightly reduced with the cut-off angle was set to 30 degree, comparing with the 15 cut-off angle results. GPS/BDS integration system position precision with the cut-off angle 30 degree is better than that of GPS/BDS standalone system with cut-off angle 15 degree.

In order to understand the time-serial results, we zoom in up component GPS/BDS standalone and integration position error serials in figure 5. Figure 6 demonstrates that not all the integration system position is optimal, comparing with the GPS/BDS standalone satellite navigation system. Epoch at 05:08:19 demonstrate BDS results is closer to zero than GPS/BDS integration system. GPS/BDS integration system reduces amplify the position error with more tracked satellites in this case.

From figure 4, all the epoch's GDOP is reduced by integrating GPS and BDS satellites. Figure 6 results find that not all the position error could be improved by simply getting involved all the tracked GPS and BDS satellites during the least square estimation. It means that small GDOP is not the sufficient indication for assessing precise positioning by integrating multi-GNSS. It is necessary to find a better indicator which can be used for predicating the positioning precision for integrating multi-GNSS system.

Table 1. GPS/BDS standalone and integration system position standard deviation at the component of north, east and up component (cut-off angle: 15/30 degree)

\begin{tabular}{cc|ccc|ccr}
\hline \multirow{2}{*}{} & & \multicolumn{3}{c|}{ cut off angle 15 } & \multicolumn{3}{c}{ cut off angle 30 } \\
\cline { 3 - 8 } & & std north & std east & std up & std north & std east & \multicolumn{1}{c}{ std up } \\
\hline \multirow{2}{*}{ GPS } & L1 & 0.893688 & 0.731707 & 2.300777 & 1.52623 & 1.302455 & 4.620561 \\
\cline { 2 - 8 } & L2 & 1.801214 & 1.438612 & 4.58488 & 2.136892 & 1.947608 & 6.5267 \\
\hline \multirow{2}{*}{ BDS } & B1 & 1.089395 & 1.101653 & 3.397337 & 2.110211 & 1.013136 & 3.647327 \\
\cline { 2 - 8 } & B2 & 0.967307 & 0.74226 & 2.463249 & 1.008995 & 0.758371 & 2.803092 \\
\hline \multirow{3}{*}{ GPS+BDS } & L1+B1 & 0.592129 & 0.625554 & 2.253816 & 0.648928 & 0.687168 & 2.3542 \\
\cline { 2 - 8 } & L2+B2 & 0.967674 & 0.797578 & 2.600986 & 0.772552 & 0.696272 & 2.433545 \\
\hline
\end{tabular}




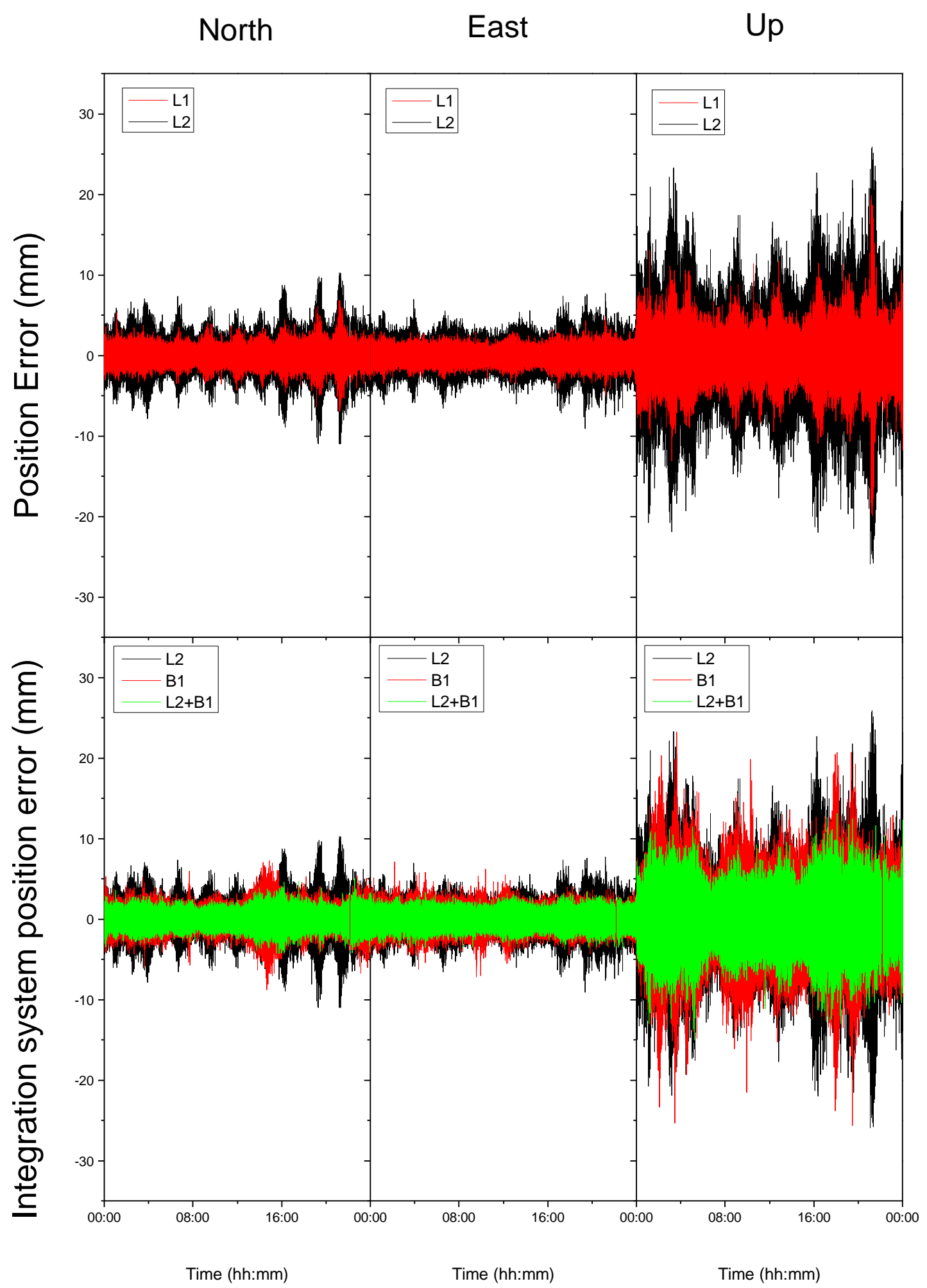

Fig 5. Comparison of GPS L1 and L2 position accuracy and the improvement of integrating GPS and BDS by L2 and B1 carrier phase measurements, respectively 


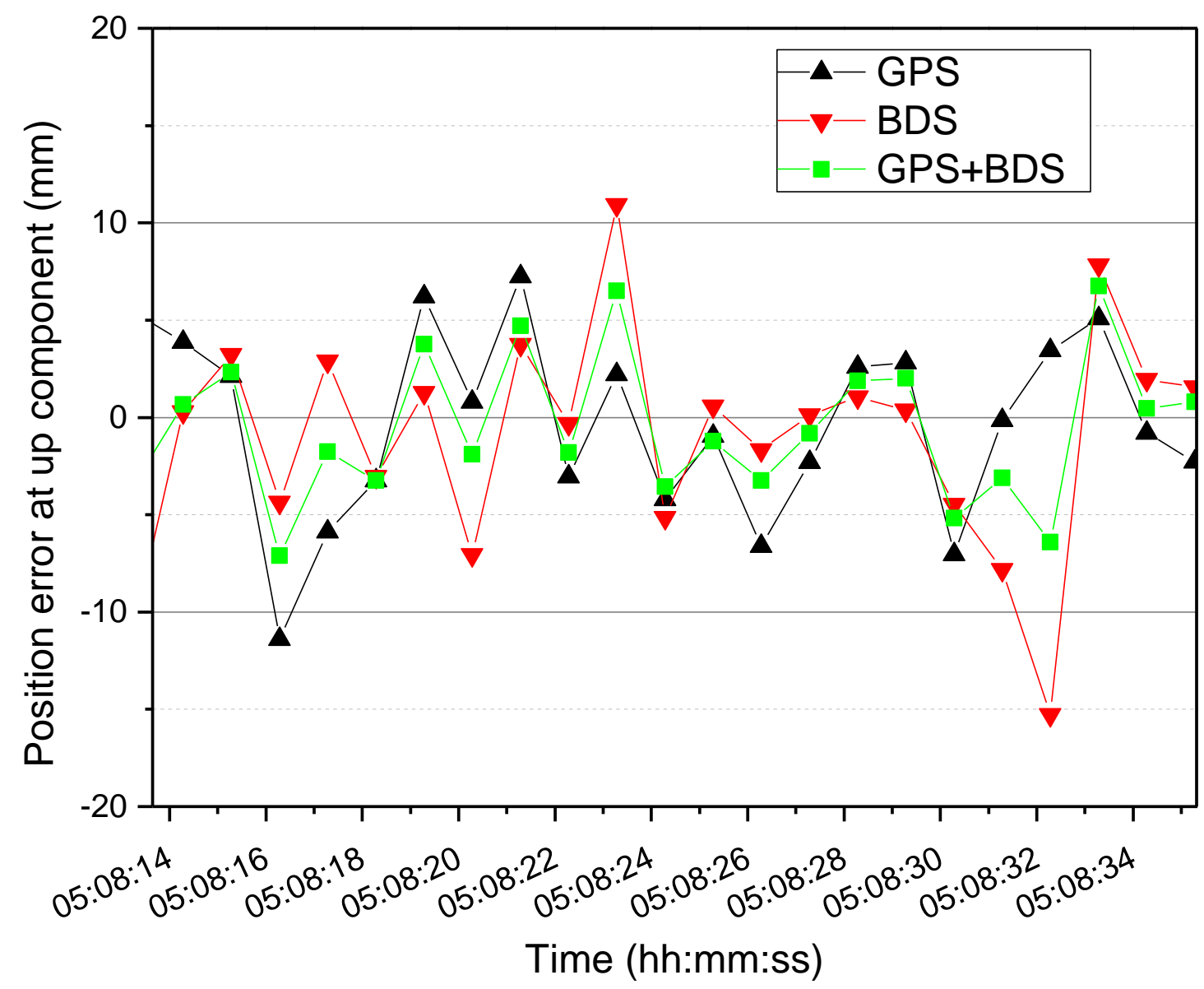

Fig. 6 detail GPS/BDS integration system position error at up component

\section{Least square estimation error amplify indicator}

The section zero baseline experiment results shown integration of GPS and BDS could get smaller GDOP, which is one of the solution for standalone system precise application with only few satellites could be tracked in some situation. Furthermore, GPS-only, BDS-only and integration of GPS and BDS positioning results are given with epoch by epoch algorithm. From the time-serial of position errors, the precision of positioning is significantly improved by integration GPS and BDS. Figure 6 gives the more detail of integration system positioning error, reveals that integration system position is not always the optimal. Meanwhile, integration GDOP is always better than standalone system. In this section, we will state how the observation equation coefficient effect the positioning precision, as well as giving an indicator for multi-GNSS integration system.

With the fixed ambiguity and approximate coordinate of antenna, zero baseline observation equation including the position correction could be derived by linearizing equation(3):

$$
\zeta=\mathbf{B X}+\mathbf{K}
$$

Where, $\zeta$ is the matrix of $\zeta_{i, j}^{p, q} ; \mathbf{X}$ is the unknown position corrections $\left[\begin{array}{lll}\delta x & \delta y & \delta z\end{array}\right]^{T}$ in general relative positioning applications. But in zero baseline experiment, 
$\left[\begin{array}{lll}\delta x & \delta y & \delta z\end{array}\right]^{T}$ is positioning errors caused by observation has noise, as the rove station is previous known, which is same with the base station. $\mathbf{B}$ is the coefficient of DD equation. With the assumption of $n$ tracked satellites and satellite 1 is the referenced satellite during double difference operator, $\mathbf{B}$ and $\mathbf{K}$ are expressed as:

$$
\mathbf{B}=\left[\begin{array}{ccc}
l_{2}^{1,2} & m_{2}^{1,2} & n_{2}^{1,2} \\
l_{2}^{1,3} & m_{2}^{1,3} & n_{2}^{1,3} \\
\vdots & \vdots & \vdots \\
l_{2}^{1, n} & m_{2}^{1, n} & n_{2}^{1, n}
\end{array}\right]_{(n-1) \times 3} \quad \mathbf{K}=\left[\begin{array}{c}
\phi_{1,2}^{1,2}-\rho_{1,2}^{1,2} \\
\phi_{1,2}^{1,3}-\rho_{1,2}^{1,3} \\
\vdots \\
\phi_{1,2}^{1, n}-\rho_{1,2}^{1, n}
\end{array}\right]_{(n-1) \times 1}
$$

$l, m, n$ in matrix $\mathbf{B}$ is the component $x, y, z$ coefficient. It should be known $l, m, n$ is calculated by the satellites coordinate under north, east and up coordinate system. $x, y, z$ is position error at components of north, east and up. $\rho$ is the Euclidian distance between satellites and the antenna. The general relationships of $\left\{\rho_{i, j}^{p, q} \mid p, q \in[1, n], i=1, j=2\right\}$ which is same with that of $g_{i, j}^{p, q}$. In zero baseline experiment, $\rho_{i, j}^{p, q}$ is the known parameter calculated by the known base station and rover station coordinates (coordinate of base and rover station is same in this case). $\phi_{i, j}^{p, q}=\varphi_{i, j}^{p, q}+N_{i, j}^{p, q}$, which means all the double differenced carrier phase measurements ambiguities are all successfully fixed. $\mathbf{K}$ is the double differenced observation random noise.

The least square correction of zero baseline could be described as

$$
\hat{\mathbf{X}}=\left(\mathbf{B}^{T} \mathbf{P B}\right)^{-1} \mathbf{B}^{T} \mathbf{P K}=\mathbf{H K}
$$

In the above equation $\mathbf{H}=\left(\mathbf{B}^{T} \mathbf{P B}\right)^{-1} \mathbf{B}^{T} \mathbf{P}$. In order to make the explanation more easier, we rewrite $\mathbf{H}$ as,

$$
\mathbf{H}=\left[\begin{array}{llll}
h_{1,1} & h_{1,2} & \cdots & h_{1,(\mathrm{n}-1)} \\
h_{2,1} & h_{2,2} & \cdots & h_{2,(\mathrm{n}-1)} \\
h_{3,1} & h_{3,2} & \cdots & h_{3,(\mathrm{n}-1)}
\end{array}\right]
$$

With the assumption of each double differenced carrier phase measurement has the same measurement error $\delta k$. The position error at the components of north, east and up could be given, respectively

$$
\begin{aligned}
& \delta x_{s y s}=h_{[1,1]_{s y s}} \delta k_{[1]_{s y s}}+h_{[1,2]_{s y s}} \delta k_{[2]_{s y s}}+\cdots+h_{\left[1,(\mathrm{n}-1)_{s y s}\right.} \delta k_{(\mathrm{n}-1)_{s y s}}=\sum_{m=1}^{n-1} h_{[1, m]_{s y s}} \delta k_{s y s} \\
& \delta y_{s y s}=h_{[2,1]_{s y s}} \delta k_{[1]_{s y s}}+h_{[2,2]_{s y s}} \delta k_{[2]_{s y s}}+\cdots+h_{[2,(\mathrm{n}-1)]_{s y s}} \delta k_{(\mathrm{n}-1)_{s y s}}=\sum_{m=1}^{n-1} h_{[2, m]_{s y s}} \delta k_{s y s} \\
& \delta z_{s y s}=h_{[3,1]_{y y s}} \delta k_{[1]_{s y s}}+h_{[3,2]_{s y s}} \delta k_{[2]_{s y s}}+\cdots+h_{\left[3,(\mathrm{n}-1]_{s y s}\right.} \delta k_{(\mathrm{n}-1)_{s y s}}=\sum_{m=1}^{n-1} h_{[3, m]_{s y s}} \delta k_{s y s}
\end{aligned}
$$


In equation (9), $\sum_{m=1}^{n-1} h_{[1, m]_{s y s}}, \sum_{m=1}^{n-1} h_{[2, m]_{s y s}}$ and $\sum_{m=1}^{n-1} h_{[3, m]_{s y s}}$ are the error amplify indicator at the component of north (x), east (y) and up (z), respectively. Subscript sys means different system, e.g. GPS, BDS or GPS/BDS integration system. Figure 7 gives 24 hours GPS-only, BDS-only and GPS/BDS integration system position error amplify indicator. Results reveals that amplify indicator at up component is bigger than north and east components by GPS-only, BDS-only, as well as GPS/BDS integration system. Then, position precision at up component is much noisier than that of north and east components in all of GPS-only, BDS-only and GPS/BDS integration system (Fig. 5), which is determined by the constellation and the observatory on earth. Figure 8 presents that, GPS/GPS integration system error amplify indicator is not optimal, comparing with the GPS and BDS standalone constellation.

GPS

BDS

GPS+BDS

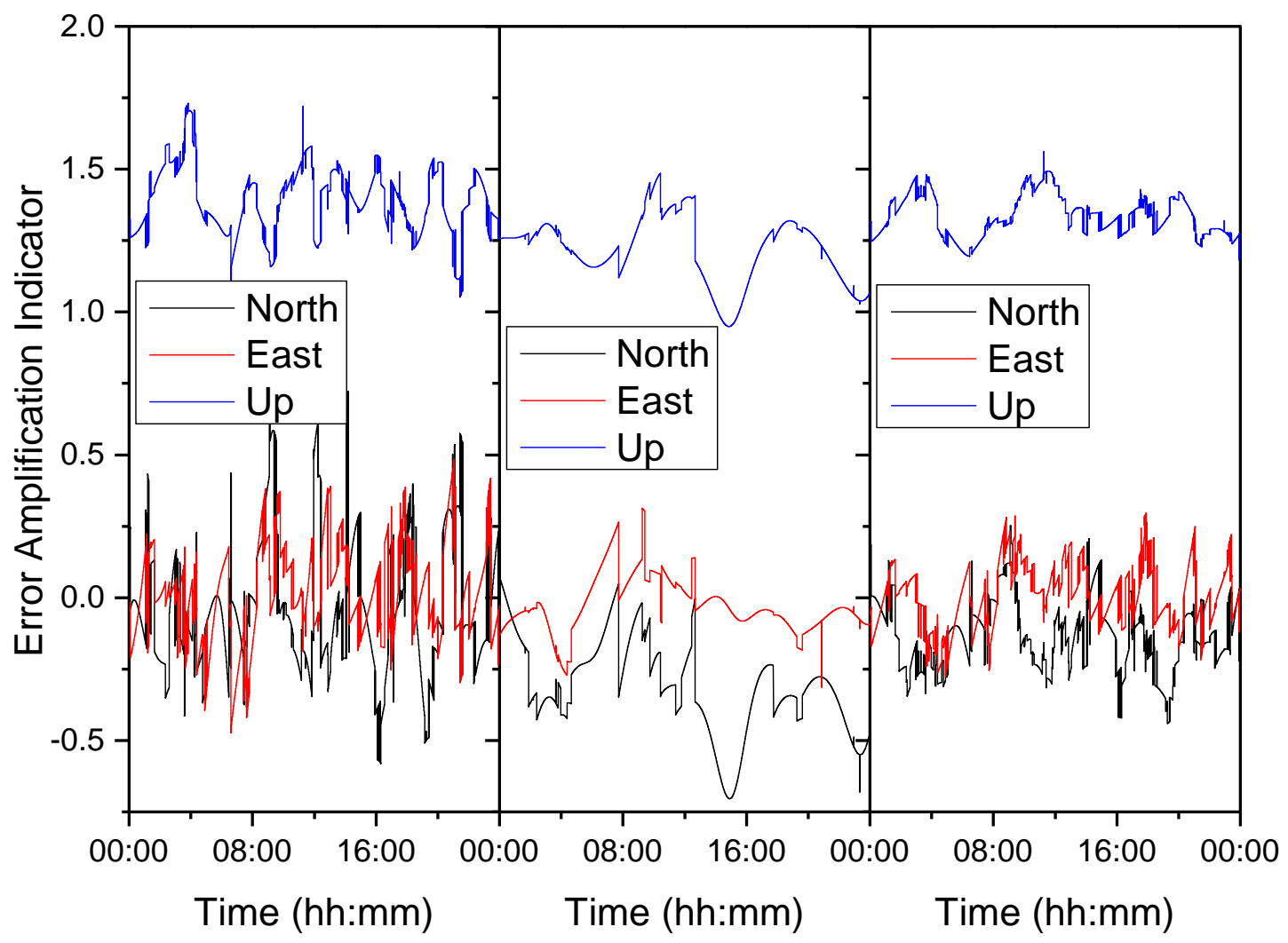

Fig. 7 GPS/BDS standalone and integration system position error amplify indicator, which is calculate by the positions of observatory and satellites

Although, GPS/BDS integration system has a bigger error amplify indicator at specific time during 24 hours, comparing with the GPS-only and BDS-only from figure 8. That does not mean integration system amplifies the position error; oppositely, figure 5 reveals GPS/BDS integration system position precision performs better than standalone system. This is because, integration system gets involved more precise carrier phase measurements. In order to prove this, with the assumption of GPS and BDS satellites have same measurement variance, GPS/BDS integration system position error variance derived from the measurements variance could be given 


$$
\begin{aligned}
& \sigma_{[x]_{s y s}}^{2}=h_{[1,1]_{s y s}}^{2} \sigma_{\left[k_{1}\right]_{s y s}}^{2}+h_{[1,2]_{y y s}}^{2} \sigma_{\left[k_{2}\right]_{y y s}}^{2}+\cdots+h_{[1,(\mathrm{n}-1)]_{s y s}}^{2} \sigma_{\left[k_{(n-1)}\right]_{s y s}}^{2}=\sum_{m=1}^{n-1} h_{[1, m]_{s y s}}^{2} \sigma^{2} \\
& \sigma_{[y]_{s y s}}^{2}=h_{[2,1]_{y y s}}^{2} \sigma_{\left[k_{1}\right]_{y y s}}^{2}+h_{[2,2]_{y y s}}^{2} \sigma_{\left[k_{2}\right]_{y y s}}^{2}+\cdots+h_{[2,(\mathrm{n}-1)]_{s y s}}^{2} \sigma_{\left[k_{(n-1)}\right]_{s y s}}^{2}=\sum_{m=1}^{n-1} h_{[2, m]_{s y s}}^{2} \sigma^{2} \\
& \sigma_{[z]_{s y s}}^{2}=h_{[3,1]_{y y s}}^{2} \sigma_{\left[k_{1}\right]_{y y s}}^{2}+h_{[3,2]_{s y s}}^{2} \sigma_{\left[k_{2}\right]_{s y s}}^{2}+\cdots+h_{\left[3,(\mathrm{n}-1]_{s y s}\right.}^{2} \sigma_{\left[k_{(n-1)}\right]_{s y s}}^{2}=\sum_{m=1}^{n-1} h_{[3, m]_{s y s}}^{2} \sigma^{2}
\end{aligned}
$$

In equation (10), $\sum_{m=1}^{n-1} h_{\{1, m]_{s y s}}^{2}, \sum_{m=1}^{n-1} h_{\{2, m]_{s y s}}^{2}$ and $\sum_{m=1}^{n-1} h_{[3, m]_{s y s}}^{2}$ are the position error variance amplify indicator at the components of north, east and up, respectively. Although GPS/BDS integration system error amplify indicator is not improved by integrating GPS and BDS system (Figure 7 and 8), the position error variance is improved by the integration system getting involved more precise double differenced carrier phase measurements (figure 9). From figure 7 to figure 9 , results reveal GPS/BDS integration system has a better position precision, this because of integration has more precise double differenced carrier phase got involved, rather than the satellites geometry is improved while the mask-off angle was set to 15 degree.

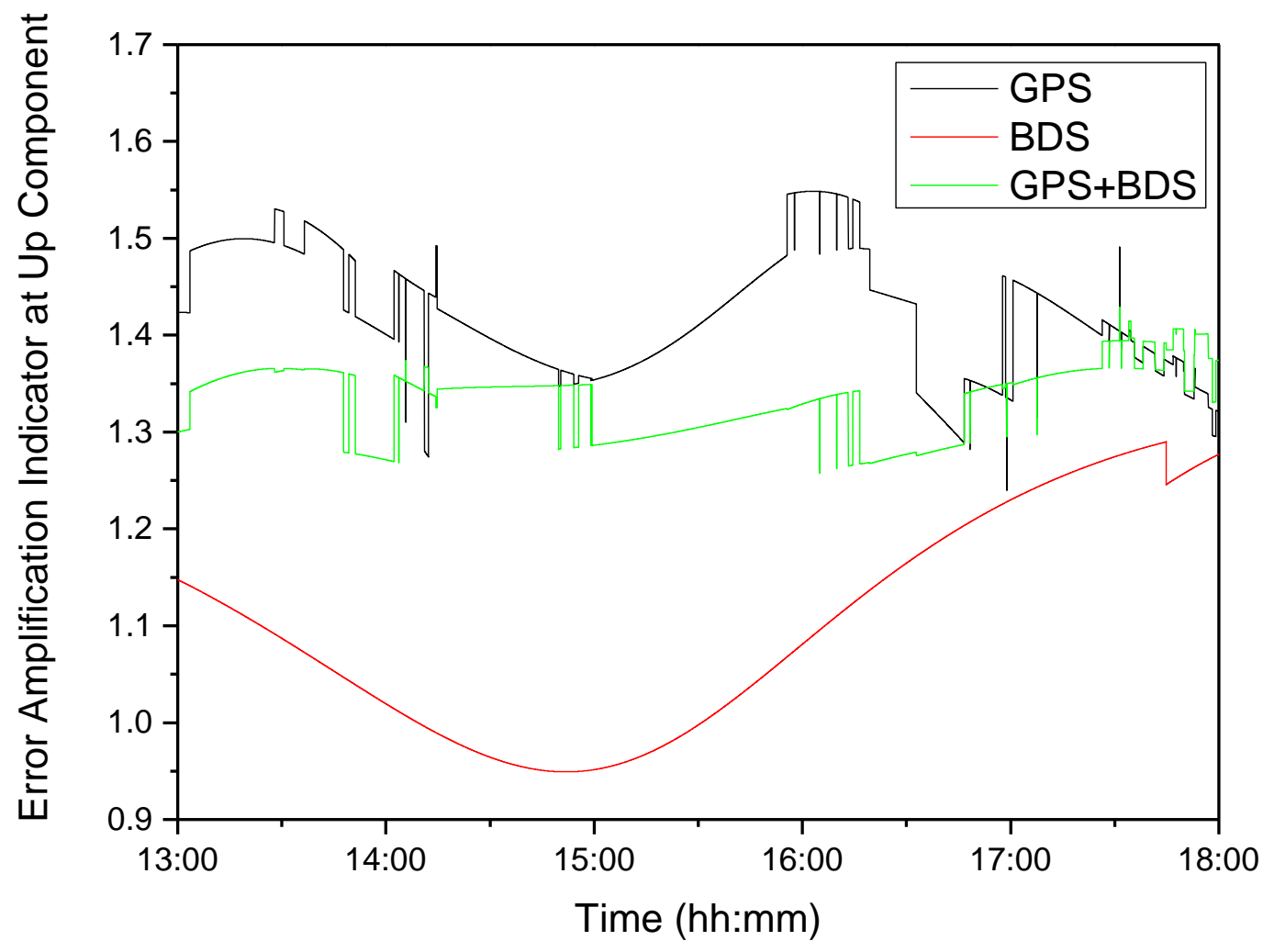

Fig. 8 comparison of GPS/BDS standalone and integration position error amplify indicator at component of up 


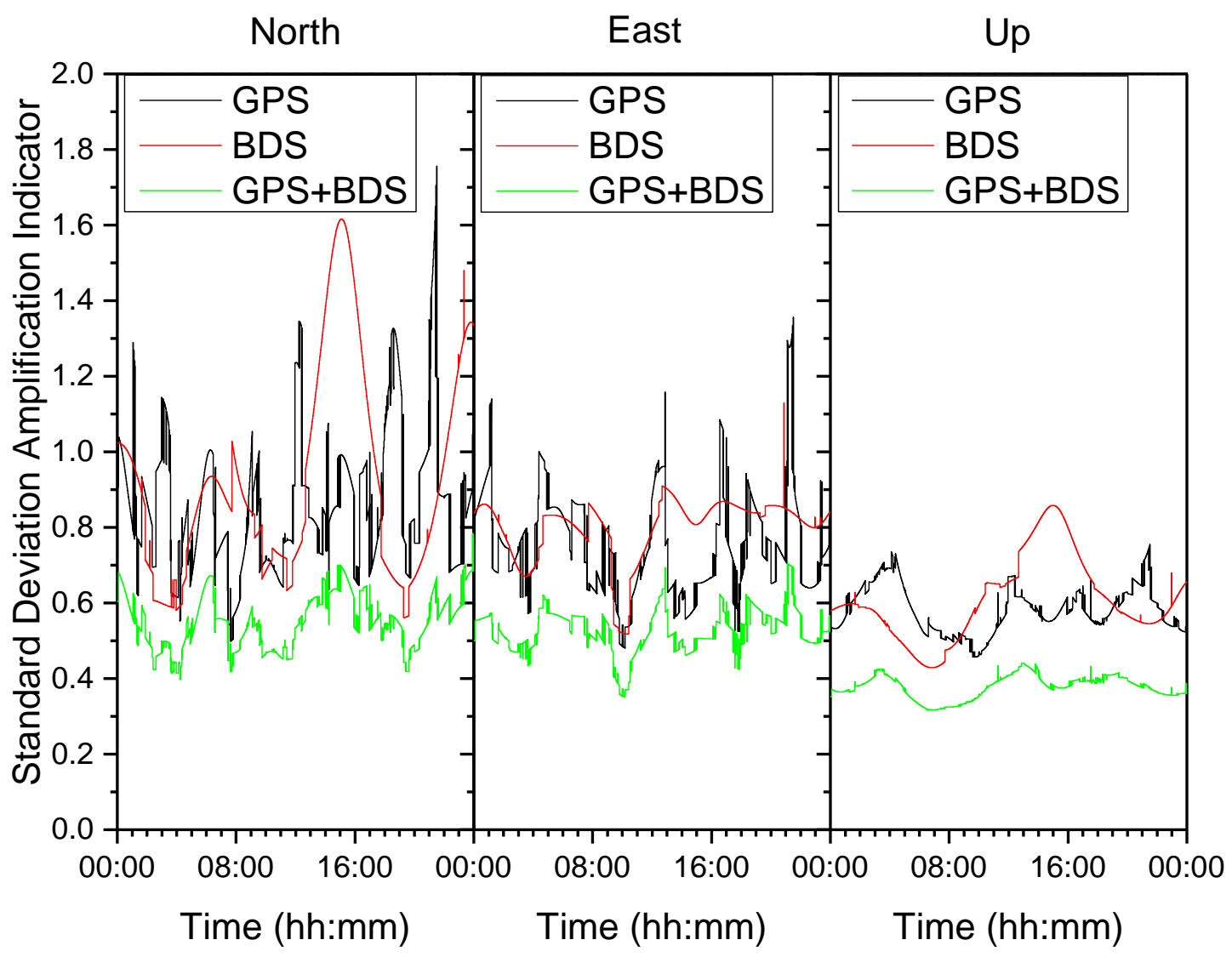

Fig. 9 GPS/BDS standalone and integration system position variance amplify indicator during 24 hours at components north, east and up

\section{Position error with different level of simulated carrier phase measurements}

The previous two sections analyzed the GPS and BDS zero baseline double differenced carrier phase measurements noise, and the position precision by GPS-only, BDS-only and GPS/BDS integration system with the cut-off elevation angle at 15 degree. Double differenced zero baseline measurements get rid of many errors which cannot be eliminated or mitigated in general baseline applications. With the only random noise left, all of GPS-only, BDS-only and GPS/BDS integration system's up component precision is worse than that of North and East component. With the GPS/BDS constellation consistence different types of satellites, it is worth to assess these constellation position precision at different level noise situations.

In this section, we simulated different levels of double differenced carrier phase measurement with the ambiguity fixed, and analyzed the standalone/integration system positioning precision with these levels of noised measurements. The " 0 " label at north/east/up components at figure 10 has only the random noise in original carrier phase measurement, variance of positioning error at component north, east and up reveals GPS performance better than BDS. Integration system gets better variance at east and up component; but only better than BDS, worse than GPS at the component of North. This result is at the extreme good situation at University of Nottingham Ningbo China. Carrier phase has much noisier in the general applications. Position error is increased with the simulated $2 \mathrm{~mm}$ step noise from $2 \mathrm{~mm}$ to $20 \mathrm{~mm}$. at the component north and east, GPS and BDS standalone system has similar precision, and BDS performs slightly better than GPS where the measurements have the increasing big noise. GPS position error increasing more 
severely than that of BDS. Integration GPS and BDS got obviously improved at different levels of noise measurement, comparing with the GPS-only and BDS-only, respectively.

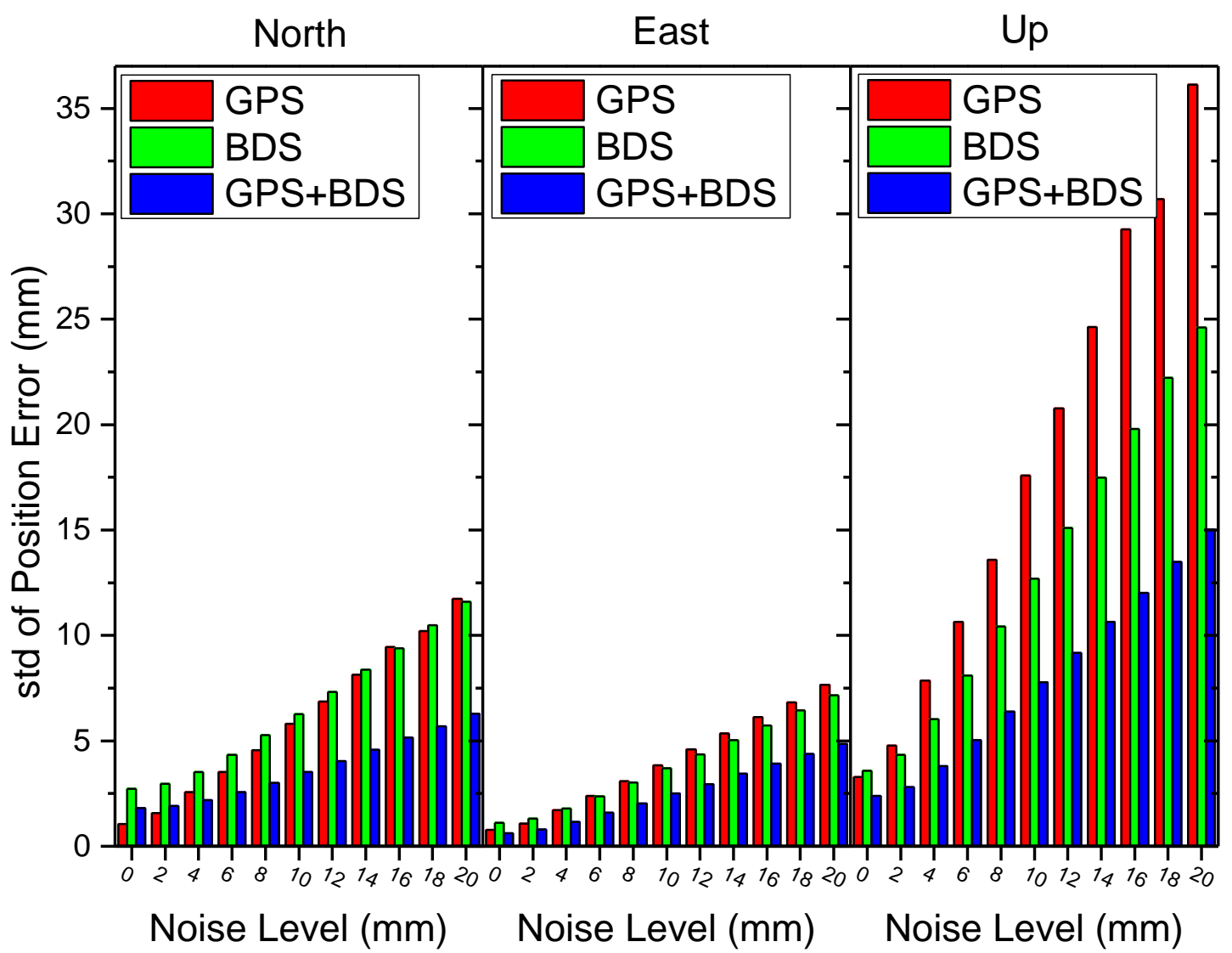

Fig. 10 Standard deviation of GPS/BDS standalone position with the different level of control simulated noise

\section{Conclusion}

GNSS Positioning precision is not only determined by the measurements precision, but also GNSS constellation itself. In this paper, we gathered 24hours GPS and BDS zero baseline measurements for assessing the GPS and BDS positioning precision. The measurement quality assessment section results reveal that, GPS L1 is more precise than its L2 and all of BDS carrier phase measurements. Furthermore, GPS positioning precision by GPS L1 carrier phase measurements is better than that of GPS L2. Precision of GPS L2 carrier phase measurements is similar with the BDS B1, B2 and B3.

GNSS GDOP is one of the indicator for assessing the quality of the constellation and the satellites distribution in previous work. In this work, we use error amplify indicator derived from the observation equations coefficient for a assessing the behavior of position error at north, east, and up component. Regards to the GPS-only, BDS-only and GPS/BDS integration system, the upcomponent positioning error is always bigger than north and east component.

Variance amplified indicator is applied to assess the integration system advantage. Although, GPS/BDS integration system error amplify indicator is not the optimal; with the help of more precise measurement getting involved, integration system achieves more precise positioning performance, comparing with the GPS/BDS standalone system.

Carrier phase measurement simulated with different levels of noise are applied to analyze 
GPS/BDS standalone and integration system position precision. GPS and BDS standalone position precision is similar at the horizontal, but GPS precision is obviously worse than BDS at the component of up. Integration GPS and BDS could significantly improve the position precision at the components of north, east and up.

This work also implies that, integration GPS/BDS system improved the positioning precision by getting more precise double differenced measurement, rather than improve the satellite geometry. In fact, integration system geometry is not the optical at some period, comparing with the standalone system.

\section{Acknowledgement}

this work is supported by Young Scientist programme of Natural Science Foundation of China with a project code 41704024 , as well as Ningbo Science and Technology Bureau as Part of the Projects: Integration of GPS and BDS to monitor large bridge on real time (2016A610116) and Structural Health Monitoring of Infrastructure in the Logistics Cycle (2014A35008).

\section{Reference}

Chen, He-Chin, Yu-Sheng Huang, Kai-Wei Chiang, Ming Yang, and Ruey-Juin Rau. 2009. "The Performance Comparison between GPs and BeiDou-2/compass: A Perspective from Asia." Journal of the Chinese Institute of Engineers 32 (5): 679-89.

Chiang, Kai-Wei, Yu-Sheng Huang, Meng-Lung Tsai, and Kwo-Hwa Chen. 2010. "The Perspective from Asia Concerning the Impact of Compass/Beidou-2 on Future GNSS." Survey Review 42 (315): 3-19. doi:10.1179/003962609X451654.

Deng, Chenlong, Weiming Tang, Jingnan Liu, and Chuang Shi. 2014. "Reliable Single-Epoch Ambiguity Resolution for Short Baselines Using Combined GPS/BeiDou System." GPS Solutions 18 (3): 375-86. doi:10.1007/s10291-013-0337-5.

Guo, Fei, Xiaohong Zhang, and Jinling Wang. 2015. "Timing Group Delay and Differential Code Bias Corrections for BeiDou Positioning." Journal of Geodesy 89 (5): 427-45. doi:10.1007/s00190-015-0788-2.

Gu, Shengfeng, Yidong Lou, Chuang Shi, and Jingnan Liu. 2015. "BeiDou Phase Bias Estimation and Its Application in Precise Point Positioning with Triple-Frequency Observable." Journal of Geodesy 89 (10): 979-92. doi:10.1007/s00190-015-0827-z.

He, Feng, ShanShi Zhou, XiaoGong Hu, JianHua Zhou, Li Liu, Rui Guo, XiaoJie Li, and Shan Wu. 2014. "Satellite-Station Time Synchronization Information Based Real-Time Orbit Error Monitoring and Correction of Navigation Satellite in Beidou System." Science China Physics, Mechanics \& Astronomy 57 (7): 1395-1403. doi:10.1007/s11433-014-5412-6.

He, Haibo, Jinlong Li, Yuanxi Yang, Junyi Xu, Hairong Guo, and Aibing Wang. 2014. "Performance Assessment of Single- and Dual-Frequency BeiDou/GPS Single-Epoch Kinematic Positioning." GPS Solutions 18 (3): 393-403. doi:10.1007/s10291-013-0339-3.

$\mathrm{Li}$, Xingxing, Maorong Ge, Xiaolei Dai, Xiaodong Ren, Mathias Fritsche, Jens Wickert, and Harald Schuh. 2015. "Accuracy and Reliability of Multi-GNSS Real-Time Precise Positioning: GPS, GLONASS, BeiDou, and Galileo." Journal of Geodesy 89 (6): 607-35. doi:10.1007/s00190015-0802-8.

Montenbruck, Oliver, André Hauschild, Peter Steigenberger, Urs Hugentobler, Peter Teunissen, and Shinichi Nakamura. 2013. "Initial Assessment of the COMPASS/BeiDou-2 Regional 
Navigation Satellite System." GPS Solutions 17 (2): 211-22. doi:10.1007/s10291-012-0272$\mathrm{x}$.

Nadarajah, Nandakumaran, Peter J. G. Teunissen, Jean-Marie Sleewaegen, and Oliver Montenbruck. 2015. "The Mixed-Receiver BeiDou Inter-Satellite-Type Bias and Its Impact on RTK Positioning." GPS Solutions 19 (3): 357-68. doi:10.1007/s10291-014-0392-6.

Odolinski, Robert, Peter J. G. Teunissen, and Dennis Odijk. 2015. "Combined BDS, Galileo, QZSS and GPS Single-Frequency RTK." GPS Solutions 19 (1): 151-63. doi:10.1007/s10291-014-03766.

Shi, Chuang, Qile Zhao, Zhigang Hu, and Jingnan Liu. 2013. "Precise Relative Positioning Using Real Tracking Data from COMPASS GEO and IGSO Satellites." GPS Solutions 17 (1): 103-19. doi:10.1007/s10291-012-0264-x.

Teunissen, P. J. G., R. Odolinski, and D. Odijk. 2014. "Instantaneous BeiDou+GPS RTK Positioning with High Cut-off Elevation Angles." Journal of Geodesy 88 (4): 335-50. doi:10.1007/s00190-013-0686-4.

Wang, Bin, Yidong Lou, Jingnan Liu, Qile Zhao, and Xing Su. 2015. "Analysis of BDS Satellite Clocks in Orbit." GPS Solutions, September. doi:10.1007/s10291-015-0488-7.

Wang, Guangxing, Kees de Jong, Qile Zhao, Zhigang Hu, and Jing Guo. 2015. "Multipath Analysis of Code Measurements for BeiDou Geostationary Satellites." GPS Solutions 19 (1): 129-39. doi:10.1007/s10291-014-0374-8.

Yang, YuanXi, JinLong Li, AiBing Wang, JunYi Xu, HaiBo He, HaiRong Guo, JunFei Shen, and Xian Dai. 2014. "Preliminary Assessment of the Navigation and Positioning Performance of BeiDou Regional Navigation Satellite System." Science China Earth Sciences 57 (1): 144-52. doi:10.1007/s11430-013-4769-0.

Ye, Shirong, Dezhong Chen, Yanyan Liu, Peng Jiang, Weiming Tang, and Pengfei Xia. 2015. "Carrier Phase Multipath Mitigation for BeiDou Navigation Satellite System." GPS Solutions 19 (4): 545-57. doi:10.1007/s10291-014-0409-1.

Yinhua, Liu, Li Xiaohui, Zhang Huijun, Zhu Feng, and Ren Ye. 2015. "Calculation and Accuracy Evaluation of TGD from IFB for BDS." GPS Solutions, April. doi:10.1007/s10291-015-04544.

Zhang, Weixing, Yidong Lou, Shengfeng Gu, Chuang Shi, Jennifer S. Haase, and Jingnan Liu. 2015. "Joint Estimation of GPS/BDS Real-Time Clocks and Initial Results." GPS Solutions, August. doi:10.1007/s10291-015-0476-y.

Zhao, Qile, Jing Guo, Min Li, Lizhong Qu, Zhigang Hu, Chuang Shi, and Jingnan Liu. 2013. "Initial Results of Precise Orbit and Clock Determination for COMPASS Navigation Satellite System." Journal of Geodesy 87 (5): 475-86. doi:10.1007/s00190-013-0622-7.

MSAEWE, H. A., HANCOCK, C. M., PSIMOULIS, P. A., ROBERTS, G. W., BONENBERG, L. \& DE LIGT, H. 2017. Investigating multi-GNSS performance in the UK and China based on a zero-baseline measurement approach. Measurement, 102, 186-199. 